\title{
Inflammatory Response, a Key Pathophysiological Mechanism of Obesity-Induced Depression
}

\author{
Shu-Lin Shao, ${ }^{1}$ Da-Qian Liu, ${ }^{2}$ Bo Zhang, ${ }^{1}$ and Shu-Ming Huang ${ }^{1}{ }^{1}$ \\ ${ }^{1}$ Department of Neuroscience, Institute for Chinese Medicine, Heilongjiang University of Chinese Medicine, Harbin, China \\ ${ }^{2}$ Department of Orthopedics, The Second Affiliated Hospital of Harbin Medical University, Harbin 150001, China \\ Correspondence should be addressed to Shu-Ming Huang; huangsm1958@126.com
}

Received 21 August 2020; Revised 17 November 2020; Accepted 18 November 2020; Published 24 November 2020

Academic Editor: Julio Galvez

Copyright (C) 2020 Shu-Lin Shao et al. This is an open access article distributed under the Creative Commons Attribution License, which permits unrestricted use, distribution, and reproduction in any medium, provided the original work is properly cited.

\begin{abstract}
In recent years, with the acceleration of life rhythm and the increase of social competition, the incidence of obesity and depression has been increasing, which has seriously affected the quality of life and health of people. Obesity and depression, two seemingly unrelated physical and psychological diseases, in fact, are closely related: obese people are more likely to have depression than nonobese ones. We have reviewed and analyzed the relevant research literature and found that the inflammatory response plays a key role in obesity-induced depression. This article will discuss in detail the inflammatory mechanisms by which obesity induces depression.
\end{abstract}

\section{Introduction}

Obesity is a disease caused by the aberrant accumulation of adipose tissue due to the imbalance between energy absorption and consumption [1], which can increase the incidence of metabolic disease, such as diabetes [2]. Depression is a serious psychiatric illness with a lifetime prevalence up to $20 \%$, whose core symptom is a lack of pleasure $[3,4]$. In severe cases, depression may lead to suicide or other negative behaviors [5], making it a major mood disorder that affects people of all ages. Recently, increasing lines of evidence have suggested that obesity increases the peril of developing depression. It has been indicated that high-fat-diet-fed mice will exhibit depressive-like behavior after several weeks' feeding $[6,7]$. Additionally, numerous clinical studies have shown that individuals who have high body mass index (BMI) are at significantly increased risk of developing depressive disorder later in life $[8,9]$.

Although it is generally agreed that obesity is associated with the onset of depression, the precise mechanisms that interactively link these two disease entities remain unclear. To address this issue, we conducted a literature search and analysis of research on experimental and clinical investigations related to depression and obesity. Interestingly, we found that when inflammatory response was assessed, raised levels of inflammatory cytokines could often be detected in the serum of populations and model animals with obesity $[10,11]$, the same was true of depressed individuals and model animals $[12,13]$. Thus, there is a strong reason to believe that inflammation is perhaps a good target to investigate how obesity affects depression. The following is a brief review of the effects of obesity on inflammation and the impacts of the inflammatory response in depression.

\section{Obesity Induces Elevation of Inflammatory Cytokines}

There is growing recognition that obesity links to inflammatory response. Epidemiological studies of individuals with obesity have demonstrated that obese people are usually accompanied by elevated levels of inflammatory cytokines. Nearly 20 years ago, to assess the association between obesity and inflammation, Ziccardi et al. carried out a comparative study on 40 nonobese and 56 obese premenopausal women, and they found in their serum the increased levels of inflammatory cytokines [14], such as tumor necrosis factor- (TNF-) $\alpha$ and interleukin- (IL-) 6. More recently, a clinical study [11] on a larger number of people in mixed-sex groups has 
reported that compared with 83 controls, in the serum, 117 obesity cases have elevated levels of tumor necrosis factor(TNF-) $\alpha$ and interleukin (IL) families or other various inflammatory mediators. Besides, the relationship between obesity and inflammatory factors has been well demonstrated in rodent models. In this regard, it is worth mentioning that the overexpressing of inflammatory cytokines in obese rodents was first discovered by Hotamisligil [15], who put forward the first molecular mechanism: tumor necrosis factor- (TNF-) $\alpha$ links obesity with inflammation. Subsequently, experimental studies have shown that the levels of inflammatory cytokines increase in obese rodents $[16,17]$ and decrease after intervention treatment $[18,19]$. And what is more, once an individual becomes obese, the mast cells' response increases, ultimately resulting in releasing massive inflammatory factors related to allergy [20,21].

\section{Mechanisms of Obesity Leading to Inflammation}

All those above strongly support an association between obesity and inflammation. It has thus become crucial to gain a better understanding of obesity-induced inflammation. As we all know, the imbalance of energy metabolism, either insufficient consumption or excessive intake, contributes to the aberrant accumulation of adipose in the body. Interestingly, the adipose tissue itself is a culprit that leads to inflammation. Adipose tissue can secrete adipokines and inflammatory factors and therefore should not be simply regarded as an energy storage or metabolic organ [22] but as an endocrine organ as well [23]. Adipocytokines, such as leptin and inflammatory cytokines, can mediate the occurrence of inflammation $[24,25]$.

Apart from the well-known adipocytes, macrophages, predominantly microglia in the brain, also play an integral role in the development of obesity-induced inflammation. It has been proved in experimental research that the migration of macrophages to adipose tissue is increased under obesity [26]. Macrophages usually have two transformed phenotypes, namely M1 macrophages and M2 macrophages [27]. The process through which obesity causes inflammation is largely attributed to M1 polarized macrophages that release proinflammatory cytokines [28]. An experimental study conducted by Kawanishi et al. has suggested that high-fatdiet-induced obesity in mice is accompanied by macrophage infiltration into adipose tissue and that suppression of macrophage infiltration or promotion of M1 to M2 macrophage conversion by experimental methods inhibits the adipose tissue inflammation [29], which has further confirmed the importance of macrophage infiltration, especially of M1 phenotype macrophages, in the process of obesity-induced inflammation.

Although the exact mechanisms responsible for obesity resulting in macrophage infiltration still elude us, there is a hypothesis accepted by most scholars that hypoxia in adipose tissue is one of the possible mechanisms of macrophage infiltration [30]. This hypothesis believes that tissue hypoxia caused by excessive fat accumulation can induce inflammation [31]. Hypoxia in adipose tissue can trigger off the inflammatory behavior of macrophages via the hypoxiainducible factor (HIF) pathway [32], ultimately leading to inflammatory response. An observational study that used high-fat-diet-induced obese mice with HIF gene knocked out and normal mice as research objects found that the obesity-induced inflammatory response was reduced after HIF gene was knocked out [33]. Furthermore, adipose tissue hypoxia can also activate the inflammation by affecting the function of the endoplasmic reticulum. An experiment carried out by Kawasaki et al. has demonstrated a strong association between endoplasmic reticulum stress and adipose tissue inflammation [34], which suggests that adipose tissue hypoxia can evoke endoplasmic reticulum stress and induce the accumulation of misfolded proteins in the endoplasmic reticulum [35] and eventually activate the inflammatory response through the action of PKR-like endoplasmic reticulum kinase (PERK) and inositol-requiring enzyme- (IRE-) 1, activating transcription factor- (ATF-) 6, and other signals [36].

\section{Inflammation Resulting in Depression}

Extensive research have proved that the inflammatory response is involved in the pathogenesis of various types of depression. Nonetheless, the precise mechanisms of inflammation affecting depression are still not fully elucidated. Over the past decades, the inadequate secretion or dysfunction of the monoaminergic neurotransmitters, including 5hydroxytryptamine (HT), norepinephrine (NE), and dopamine (DA) [37], has been considered to have the most direct relationship with the onset of depression. This opinion has been attested in numerous clinical and experimental studies [38-40]. Some subsequent studies have reported that there are some shortcomings in the traditional monoaminergic transmitter theory, which make it difficult to explain some phenomena in the research process of depression. For example, antidepressant drugs, which were developed based on this principle and can increase the neurotransmitter of the synaptic space of monoaminergic neurons, can elevate the concentration of monoaminergic neurotransmitters in the synaptic gaps of related neurons in the brain in a short period. However, it takes 1-2 weeks to become effective in clinical practice [41]. Recently, several new hypotheses on the pathogenesis of depression, such as neuronal injury, abnormal neurogenesis, and excessive activation of glial cells, have been put forward and postulated to link with monoaminergic neurotransmitters [42-44].

Neuronal damage, degenerative disorders, or neurogenesis obstacles can consistently induce the deficiencies of these mood-related neurotransmitters $[45,46]$. Similarly, the activation of glial cells in the brain may decrease the concentration of monoaminergic neurotransmitters near the synaptic cleft through excessive uptake of the transmitters [47]. Thus, neuronal injury, neurogenesis obstacles, and glial cell overactivation are viewed as potential mechanisms to induce depression [48-50], because they play vital roles in affecting the concentration of monoaminergic transmitters in the synaptic cleft. This viewpoint has been proved in various experimental studies in which animal models of depression 
replicated by chronic unpredictable mild stimulation (CUMS) were usually accompanied by abnormal neurogenesis, neuronal damage, and glial cell hyperactivation [5153]. Likewise, these phenomena could be alleviated or reversed by antidepression drug treatment [53-55].

Interestingly, the inflammatory response almost affects all those above mechanisms. Numerous researches have manifested that the inflammatory cytokines can impose effects on monoaminergic neurotransmitters [56-59]. For instance, an experiment conducted by Kaster et al. has shown that the injection of tumor necrosis factor- $\alpha$ (TNF- $\alpha)$ into the mice may cause a depressive-like behavior [60]. This phenomenon may be induced by the activation of indoleamine 2,3-dioxygenase (IDO) in the peripheral (mainly hepatic), and the conversion of tryptophan (Trp) into kynurenic acid (Kyn), thus affecting the normal secretion of 5-HT [61, 62]. Furthermore, the inflammatory response may cause neurogenesis disorders. This theory has been proved in a related experimental study, in which it has been found that the lipopolysaccharide- (LPS-) induced inflammation in the brain might play a key role in inhibiting the process of neuronal neogenesis from neural stem cells to neurons [63]. When inflammation occurs in the brain, activated M1 phenotype microglia is the main culprit in inhibiting neuronal neogenesis, while M2 phenotype microglia which has antiinflammatory effect is beneficial to neurogenesis. A study reported that pioglitazone alleviated maternal sleep deprivation induced cognitive deficits in rat offspring by enhancing neurogenesis through switching microglial phenotype from M1 to M2 [64].

Also, the inflammatory reaction may induce neuronal damage. Hoffmann et al. have carried out experimental research, in which the phenomenon of neuronal injury can be observed during Toll-like receptor 2- (TLR2-) mediated neuroinflammation [65]. Besides, the inflammation may play a critical role in stimulating the activation of glial cells. It has been manifested in an experiment report that the synergistic effect of Toll-like receptor 4 (TLR4) and reactive oxygen species (ROS) can mediate the activation of glial cells in lipopolysaccharide- (LPS-) induced inflammatory response, thereby releasing a large number of inflammatory cytokines [66]. An experimental study indicated that inhibiting microglial activation in the brain could alleviate depressive-like behaviors in adolescent mice subjected to maternal separation [67].

It should be noted that obesity may lead to some changes in the permeability of the blood-brain barrier, resulting in obesity-related metabolites and inflammatory factors, such as free fatty acids and IL-6, and even some immune cells, more likely to enter the central nervous system [68]. In obese individuals, not only inflammatory factors but also obesityrelated metabolites increase in the blood. In addition to the peripheral inflammatory factors, some peripheral obesityrelated metabolites, such as free fatty acid, can also stimulate the central immune cells to release inflammatory factors after they enter the central nervous system [69].

Besides, it should also be added that increasing evidence has shown that obese people often have various degrees of intestinal microbiota imbalance [70-72]. Some dysbacterio- sis can induce depression by affecting the metabolism of protein or fat and the secretion of digestive tract hormones and central hormones $[73,74]$. Recent studies suggest that animal models implanted with the gut microbiota of depressive people are more likely to be depressed $[75,76]$. Moreover, there is emerging data that depression can be treated by regulating gut microbiota $[77,78]$. These findings raise the possibility that gut microbiota plays a role in the depression of obesity people with intestinal microbiota imbalance. Yet, it must be pointed out that the causal relationship between obesity and intestinal microbiota is currently uncertain. Although there are a few reports that obesity can change intestinal microbiota, growing studies support the role of gut microbiome in the development of obesity. Intestinal dysbacteriosis can lead to an increase in energy obtained from food or promote energy metabolism to affect energy balance [79, 80 ], which can inevitably result in obesity. There is ample evidence that regulating intestinal microbiota can reduce the weight of such people $[81,82]$. Therefore, in those obesityinduced depression people who have intestinal microbiota imbalance, the intestinal dysbacteriosis may be an initial mechanism through which depression occurs.

Intestinal microbiota imbalance is a complex concept. Many kinds of bacteria parasitize in intestine, and their imbalance does not always induce obesity or depression. Whether the dysbacteriosis (causes obesity or caused by obesity) leads to depression through gut-brain axis needs to be studied further.

The imbalance of intestinal microbiota may influence the function of digestive tract mucosa, which thereby can absorb some small molecules of inflammatory substances, placing the body into a subclinical inflammatory status [83]. Therefore, the imbalance of intestinal microbiota can promote the occurrence of depression through the aforementioned central inflammatory mechanisms, and we cannot ignore the role of inflammation in the process of gut microbiota related depression [84].

\section{Conclusion}

This review took the inflammatory response as an entry point to explore the relationship between obesity and depression. After analyzing recent relevant studies, we presented a new perspective on the occurrence and development of obesityinduced depression: the inflammatory response is a key pathophysiological mechanism of the disorder.

\section{Additional Points}

Highlights. (1) Obesity may induce inflammatory response. (2) Some peripheral inflammatory factors can cross the blood-brain barrier and cause inflammatory response in the brain. (3) The cerebral inflammatory response may cause depression through the mechanisms of neuronal injury, excessive activation of glial cells, and hindering neurogenesis.

\section{Conflicts of Interest}

No potential conflict of interest was reported by the authors. 


\section{Acknowledgments}

This work was supported by the National Natural Science Foundation of China (No. 81873108), University Nursing Program for Young Scholars with Creative Talents in Heilongjiang Province (No. UNPYSCT-2017216), and Research Foundation of Heilongjiang University of Chinese Medicine (No. 2019BJP02).

\section{References}

[1] B. M. Spiegelman and J. S. Flier, "Obesity and the regulation of energy balance,” Cell, vol. 104, no. 4, pp. 531-543, 2001.

[2] A. Hruby, J. A. E. Manson, L. Qi et al., "Determinants and consequences of obesity," American Journal of Public Health, vol. 106, no. 9, pp. 1656-1662, 2016.

[3] D. E. Battle, "Diagnostic and statistical manual of mental disorders (DSM)," Codas, vol. 25, no. 2, pp. 191-192, 2013.

[4] C. Menard, G. E. Hodes, and S. J. Russo, "Pathogenesis of depression: insights from human and rodent studies," Neuroscience, vol. 321, pp. 138-162, 2016.

[5] K. Hawton, C. C. i Comabella, C. Haw, and K. Saunders, "Risk factors for suicide in individuals with depression: a systematic review," Journal of Affective Disorders, vol. 147, no. 1-3, pp. 17-28, 2013.

[6] E. Vagena, J. K. Ryu, B. Baeza-Raja et al., "A high-fat diet promotes depression-like behavior in mice by suppressing hypothalamic PKA signaling," Translational Psychiatry, vol. 9, no. 1, p. 141, 2019.

[7] S. Sharma and S. Fulton, "Diet-induced obesity promotes depressive-like behaviour that is associated with neural adaptations in brain reward circuitry," International Journal of Obesity, vol. 37, no. 3, pp. 382-389, 2013.

[8] J. Tyrrell, A. Mulugeta, A. R. Wood et al., "Using genetics to understand the causal influence of higher BMI on depression," International Journal of Epidemiology, vol. 48, no. 3, pp. 834$848,2019$.

[9] C. F. Hung, M. Rivera, N. Craddock et al., "Relationship between obesity and the risk of clinically significant depression: Mendelian randomisation study," The British Journal of Psychiatry, vol. 205, no. 1, pp. 24-28, 2014.

[10] M. Ladefoged, K. Buschard, and A. M. K. Hansen, "Increased expression of toll-like receptor 4 and inflammatory cytokines, interleukin- 6 in particular, in islets from a mouse model of obesity and type 2 diabetes," APMIS, vol. 121, no. 6, pp. 531538, 2013.

[11] F. M. Schmidt, J. Weschenfelder, C. Sander et al., "Inflammatory cytokines in general and central obesity and modulating effects of physical activity," PLoS One, vol. 10, no. 3, article e0121971, 2015.

[12] Z. You, C. Luo, W. Zhang et al., "Pro- and anti-inflammatory cytokines expression in rat's brain and spleen exposed to chronic mild stress: involvement in depression," Behavioural Brain Research, vol. 225, no. 1, pp. 135-141, 2011.

[13] Y. He, W. Li, Y. Wang et al., "Major depression accompanied with inflammation and multiple cytokines alterations: evidences from clinical patients to Macaca fascicularis and LPSinduced depressive mice model," Journal of Affective Disorders, vol. 271, pp. 262-271, 2020.

[14] P. Ziccardi, F. Nappo, G. Giugliano et al., "Reduction of inflammatory cytokine concentrations and improvement of endothelial functions in obese women after weight loss over one year," Circulation, vol. 105, no. 7, pp. 804-809, 2002.

[15] G. S. Hotamisligil, N. S. Shargill, and B. M. Spiegelman, "Adipose expression of tumor necrosis factor-alpha: direct role in obesity-linked insulin resistance," Science, vol. 259, no. 5091, pp. 87-91, 1993.

[16] Z. Liu, R. S. Brooks, E. D. Ciappio et al., "Diet-induced obesity elevates colonic TNF-alpha in mice and is accompanied by an activation of Wnt signaling: a mechanism for obesityassociated colorectal cancer," The Journal of Nutritional Biochemistry, vol. 23, no. 10, pp. 1207-1213, 2012.

[17] K. Boonloh, V. Kukongviriyapan, B. Kongyingyoes, U. Kukongviriyapan, S. Thawornchinsombut, and P. Pannangpetch, "Rice bran protein Hydrolysates improve insulin resistance and decrease pro-inflammatory cytokine gene expression in rats fed a high carbohydrate-high fat diet," Nutrients, vol. 7, no. 8, pp. 6313-6329, 2015.

[18] S. Kim, Y. Jin, Y. Choi, and T. Park, "Resveratrol exerts antiobesity effects via mechanisms involving down-regulation of adipogenic and inflammatory processes in mice," Biochemical Pharmacology, vol. 81, no. 11, pp. 1343-1351, 2011.

[19] N. Kawanishi, H. Yano, T. Mizokami, M. Takahashi, E. Oyanagi, and K. Suzuki, "Exercise training attenuates hepatic inflammation, fibrosis and macrophage infiltration during diet induced-obesity in mice," Brain, Behavior, and Immunity, vol. 26, no. 6, pp. 931-941, 2012.

[20] A. Divoux, S. Moutel, C. Poitou et al., "Mast cells in human adipose tissue: link with morbid obesity, inflammatory status, and diabetes," The Journal of Clinical Endocrinology and Metabolism, vol. 97, no. 9, pp. E1677-E1685, 2012.

[21] P. Żelechowska, J. Agier, E. Kozłowska, and E. BrzezińskaBłaszczyk, "Mast cells participate in chronic low-grade inflammation within adipose tissue," Obesity Reviews, vol. 19, no. 5, pp. 686-697, 2018.

[22] S. S. Choe, J. Y. Huh, I. J. Hwang, J. In Kim, and J. B. Kim, "Adipose tissue remodeling: its role in energy metabolism and metabolic disorders," Frontiers in Endocrinology, vol. 7, p. 30, 2016.

[23] S. Galic, J. S. Oakhill, and G. R. Steinberg, "Adipose tissue as an endocrine organ," Molecular and Cellular Endocrinology, vol. 316, no. 2, pp. 129-139, 2010.

[24] Y. F. Tian, W. C. Chang, C. H. Loh, and P. S. Hsieh, "Leptinmediated inflammatory signaling crucially links visceral fat inflammation to obesity-associated beta-cell dysfunction," Life Sciences, vol. 116, no. 1, pp. 51-58, 2014.

[25] C. Lagathu, J. P. Bastard, M. Auclair, M. Maachi, J. Capeau, and M. Caron, "Chronic interleukin-6 (IL-6) treatment increased IL-6 secretion and induced insulin resistance in adipocyte: prevention by rosiglitazone," Biochemical and Biophysical Research Communications, vol. 311, no. 2, pp. 372-379, 2003.

[26] D. Y. Oh, H. Morinaga, S. Talukdar, E. J. Bae, and J. M. Olefsky, "Increased macrophage migration into adipose tissue in obese mice," Diabetes, vol. 61, no. 2, pp. 346-354, 2012.

[27] S. Fujisaka, I. Usui, A. Bukhari et al., "Regulatory mechanisms for adipose tissue M1 and M2 macrophages in diet-induced obese mice," Diabetes, vol. 58, no. 11, pp. 2574-2582, 2009.

[28] C. N. Lumeng, J. L. Bodzin, and A. R. Saltiel, "Obesity induces a phenotypic switch in adipose tissue macrophage polarization," The Journal of Clinical Investigation, vol. 117, no. 1, pp. 175-184, 2007. 
[29] N. Kawanishi, H. Yano, Y. Yokogawa, and K. Suzuki, "Exercise training inhibits inflammation in adipose tissue via both suppression of macrophage infiltration and acceleration of phenotypic switching from M1 to M2 macrophages in high-fat-dietinduced obese mice," Exercise Immunology Review, vol. 16, pp. 105-118, 2010.

[30] L. K. Heilbronn and L. V. Campbell, “Adipose tissue macrophages, low grade inflammation and insulin resistance in human obesity," Current Pharmaceutical Design, vol. 14, no. 12, pp. 1225-1230, 2008.

[31] M. E. Rausch, S. Weisberg, P. Vardhana, and D. V. Tortoriello, "Obesity in C57BL/6J mice is characterized by adipose tissue hypoxia and cytotoxic T-cell infiltration," International Journal of Obesity, vol. 32, no. 3, pp. 451-463, 2008.

[32] S. Fujisaka, I. Usui, M. Ikutani et al., "Adipose tissue hypoxia induces inflammatory M1 polarity of macrophages in an HIF-1alpha-dependent and HIF-1alpha-independent manner in obese mice," Diabetologia, vol. 56, no. 6, pp. 1403-1412, 2013.

[33] Y. Kihira, M. Miyake, M. Hirata et al., "Deletion of hypoxiainducible factor-1alpha in adipocytes enhances glucagon-like peptide- 1 secretion and reduces adipose tissue inflammation," PLoS One, vol. 9, no. 4, article e93856, 2014.

[34] N. Kawasaki, R. Asada, A. Saito, S. Kanemoto, and K. Imaizumi, "Obesity-induced endoplasmic reticulum stress causes chronic inflammation in adipose tissue," Scientific Reports, vol. 2, no. 1, p. 799, 2012.

[35] E. Yilmaz, "Endoplasmic reticulum stress and obesity," Advances in Experimental Medicine and Biology, vol. 960, pp. 261-276, 2017.

[36] J. Grootjans, A. Kaser, R. J. Kaufman, and R. S. Blumberg, "The unfolded protein response in immunity and inflammation," Nature Reviews. Immunology, vol. 16, no. 8, pp. 469-484, 2016.

[37] M. Hamon and P. Blier, "Monoamine neurocircuitry in depression and strategies for new treatments," Progress in Neuro-Psychopharmacology \& Biological Psychiatry, vol. 45, pp. 54-63, 2013.

[38] J. P. R. Jacobsen, W. B. Siesser, B. D. Sachs et al., "Deficient serotonin neurotransmission and depression-like serotonin biomarker alterations in tryptophan hydroxylase 2 (Tph2) loss-of-function mice," Molecular Psychiatry, vol. 17, no. 7, pp. 694-704, 2012.

[39] B. Haenisch, A. Bilkei-Gorzo, M. G. Caron, and H. Bönisch, "Knockout of the norepinephrine transporter and pharmacologically diverse antidepressants prevent behavioral and brain neurotrophin alterations in two chronic stress models of depression," Journal of Neurochemistry, vol. 111, no. 2, pp. 403-416, 2009.

[40] H. G. Ruhe, N. S. Mason, and A. H. Schene, "Mood is indirectly related to serotonin, norepinephrine and dopamine levels in humans: a meta-analysis of monoamine depletion studies," Molecular Psychiatry, vol. 12, no. 4, pp. 331-359, 2007.

[41] A. A. Nierenberg, A. H. Farabaugh, J. E. Alpert et al., "Timing of onset of antidepressant response with fluoxetine treatment," The American Journal of Psychiatry, vol. 157, no. 9, pp. 14231428, 2000.

[42] M. R. Islam, S. Moriguchi, H. Tagashira, and K. Fukunaga, "Rivastigmine improves hippocampal neurogenesis and depression-like behaviors via 5-HT1A receptor stimulation in olfactory bulbectomized mice," Neuroscience, vol. 272, pp. 116-130, 2014.
[43] C. Schaper, Y. Zhu, M. Kouklei, C. Culmsee, and J. Krieglstein, "Stimulation of 5-HT(1A) receptors reduces apoptosis after transient forebrain ischemia in the rat," Brain Research, vol. 883, no. 1, pp. 41-50, 2000.

[44] C. G. Schipke, I. Heuser, and O. Peters, "Antidepressants act on glial cells: SSRIs and serotonin elicit astrocyte calcium signaling in the mouse prefrontal cortex," Journal of Psychiatric Research, vol. 45, no. 2, pp. 242-248, 2011.

[45] M. Hinz, A. Stein, and T. Uncini, "Relative nutritional deficiencies associated with centrally acting monoamines," International Journal of General Medicine, vol. 5, pp. 413-430, 2012.

[46] Z. Kohl, N. Ben Abdallah, J. Vogelgsang et al., "Severely impaired hippocampal neurogenesis associates with an early serotonergic deficit in a BAC alpha-synuclein transgenic rat model of Parkinson's disease," Neurobiology of Disease, vol. 85, pp. 206-217, 2016.

[47] A. Castaño, A. J. Herrera, J. Cano, and A. Machado, "Lipopolysaccharide intranigral injection induces inflammatory reaction and damage in nigrostriatal dopaminergic system," Journal of Neurochemistry, vol. 70, no. 4, pp. 1584-1592, 1998.

[48] J. S. Snyder, A. Soumier, M. Brewer, J. Pickel, and H. A. Cameron, "Adult hippocampal neurogenesis buffers stress responses and depressive behaviour," Nature, vol. 476, no. 7361, pp. 458461, 2011.

[49] M. M. C. Gonzalez and G. Aston-Jones, "Light deprivation damages monoamine neurons and produces a depressive behavioral phenotype in rats," Proceedings of the National Academy of Sciences of the United States of America, vol. 105, no. 12, pp. 4898-4903, 2008.

[50] D. J. Stein, M. F. Vasconcelos, L. Albrechet-Souza, K. M. M. Ceresér, and R. M. M. de Almeida, "Microglial overactivation by social defeat stress contributes to anxiety- and depressive-like behaviors," Frontiers in Behavioral Neuroscience, vol. 11, p. 207, 2017.

[51] K. J. Lee, S. J. Kim, S. W. Kim et al., "Chronic mild stress decreases survival, but not proliferation, of new-born cells in adult rat hippocampus," Experimental \& Molecular Medicine, vol. 38, no. 1, pp. 44-54, 2006.

[52] A. M. Magariños, B. S. McEwen, G. Flügge, and E. Fuchs, "Chronic psychosocial stress causes apical dendritic atrophy of hippocampal CA3 pyramidal neurons in subordinate tree shrews," The Journal of Neuroscience, vol. 16, no. 10, pp. 3534-3540, 1996.

[53] R. K. Farooq, A. Tanti, S. Ainouche, S. Roger, C. Belzung, and V. Camus, "A P2X7 receptor antagonist reverses behavioural alterations, microglial activation and neuroendocrine dysregulation in an unpredictable chronic mild stress (UCMS) model of depression in mice," Psychoneuroendocrinology, vol. 97, pp. 120-130, 2018.

[54] Z. Kohl, B. Winner, K. Ubhi et al., "Fluoxetine rescues impaired hippocampal neurogenesis in a transgenic A53T synuclein mouse model," The European Journal of Neuroscience, vol. 35, no. 1, pp. 10-19, 2012.

[55] K. Kobayashi, Y. Ikeda, A. Sakai et al., "Reversal of hippocampal neuronal maturation by serotonergic antidepressants," Proceedings of the National Academy of Sciences of the United States of America, vol. 107, no. 18, pp. 8434-8439, 2010.

[56] Y. Couch, C. J. Martin, C. Howarth et al., "Systemic inflammation alters central 5-HT function as determined by pharmacological MRI," NeuroImage, vol. 75, no. 100, pp. 177-186, 2013. 
[57] S. van den Ameele, D. Fuchs, V. Coppens et al., "Markers of inflammation and monoamine metabolism indicate accelerated aging in bipolar disorder," Frontiers in Psychiatry, vol. 9, p. $250,2018$.

[58] F. E. Lotrich, "Inflammatory cytokine-associated depression," Brain Research, vol. 1617, pp. 113-125, 2015.

[59] M. E. Bauer and A. L. Teixeira, "Inflammation in psychiatric disorders: what comes first?," Annals of the New York Academy of Sciences, vol. 1437, no. 1, pp. 57-67, 2019.

[60] M. P. Kaster, V. M. Gadotti, J. B. Calixto, A. R. S. Santos, and A. L. S. Rodrigues, "Depressive-like behavior induced by tumor necrosis factor- $\alpha$ in mice," Neuropharmacology, vol. 62, no. 1, pp. 419-426, 2012.

[61] M. C. Wichers and M. Maes, "The role of indoleamine 2,3dioxygenase (IDO) in the pathophysiology of interferonalpha-induced depression," Journal of Psychiatry \& Neuroscience, vol. 29, no. 1, pp. 11-17, 2004.

[62] M. Maes, B. E. Leonard, A. M. Myint, M. Kubera, and R. Verkerk, "The new '5-HT' hypothesis of depression: cellmediated immune activation induces indoleamine 2,3-dioxygenase, which leads to lower plasma tryptophan and an increased synthesis of detrimental tryptophan catabolites (TRYCATs), both of which contribute to the onset of depression," Progress in Neuro-Psychopharmacology \& Biological Psychiatry, vol. 35, no. 3, pp. 702-721, 2011.

[63] A. Mouihate, "TLR4-mediated brain inflammation halts neurogenesis: impact of hormonal replacement therapy," Frontiers in Cellular Neuroscience, vol. 8, p. 146, 2014.

[64] Y. Han, J. Wang, Q. Zhao et al., "Pioglitazone alleviates maternal sleep deprivation-induced cognitive deficits in male rat offspring by enhancing microglia-mediated neurogenesis," Brain, Behavior, and Immunity, vol. 87, pp. 568-578, 2020.

[65] O. Hoffmann, J. S. Braun, D. Becker et al., "TLR2 mediates neuroinflammation and neuronal damage," Journal of Immunology, vol. 178, no. 10, pp. 6476-6481, 2007.

[66] L. Qin, G. Li, X. Qian et al., "Interactive role of the toll-like receptor 4 and reactive oxygen species in LPS-induced microglia activation," Glia, vol. 52, no. 1, pp. 78-84, 2005.

[67] Y. Han, L. Zhang, Q. Wang et al., "Minocycline inhibits microglial activation and alleviates depressive-like behaviors in male adolescent mice subjected to maternal separation," Psychoneuroendocrinology, vol. 107, pp. 37-45, 2019.

[68] E. M. Rhea, T. S. Salameh, A. F. Logsdon, A. J. Hanson, M. A. Erickson, and W. A. Banks, "Blood-brain barriers in obesity," The AAPS Journal, vol. 19, no. 4, pp. 921-930, 2017.

[69] A. A. Miller and S. J. Spencer, "Obesity and neuroinflammation: a pathway to cognitive impairment," Brain, Behavior, and Immunity, vol. 42, pp. 10-21, 2014.

[70] R. E. Ley, F. Backhed, P. Turnbaugh, C. A. Lozupone, R. D. Knight, and J. I. Gordon, "Obesity alters gut microbial ecology," Proceedings of the National Academy of Sciences of the United States of America, vol. 102, no. 31, pp. 11070-11075, 2005.

[71] P. J. Turnbaugh, R. E. Ley, M. A. Mahowald, V. Magrini, E. R. Mardis, and J. I. Gordon, "An obesity-associated gut microbiome with increased capacity for energy harvest," Nature, vol. 444, no. 7122, pp. 1027-1031, 2006.

[72] A. Koliada, G. Syzenko, V. Moseiko et al., "Association between body mass index and Firmicutes/Bacteroidetes ratio in an adult Ukrainian population," BMC Microbiology, vol. 17 , no. 1 , p. $120,2017$.
[73] S. A. Joyce, J. MacSharry, P. G. Casey et al., "Regulation of host weight gain and lipid metabolism by bacterial bile acid modification in the gut," Proceedings of the National Academy of Sciences of the United States of America, vol. 111, no. 20, pp. 7421-7426, 2014.

[74] M. Soto, C. Herzog, J. A. Pacheco et al., "Gut microbiota modulate neurobehavior through changes in brain insulin sensitivity and metabolism," Molecular Psychiatry, vol. 23, no. 12, pp. 2287-2301, 2018.

[75] P. Zheng, B. Zeng, C. Zhou et al., "Gut microbiome remodeling induces depressive-like behaviors through a pathway mediated by the host's metabolism," Molecular Psychiatry, vol. 21, no. 6, pp. 786-796, 2016.

[76] J. R. Kelly, Y. Borre, C. O'Brien et al., “Transferring the blues: Depression-associated gut microbiota induces neurobehavioural changes in the rat," Journal of Psychiatric Research, vol. 82, pp. 109-118, 2016.

[77] H. Z. Zhu, Y. D. Liang, Q. Y. Ma et al., "Xiaoyaosan improves depressive-like behavior in rats with chronic immobilization stress through modulation of the gut microbiota," Biomedicine \& Pharmacotherapy, vol. 112, p. 108621, 2019.

[78] Y. Sun, W. Geng, Y. Pan, J. Wang, P. Xiao, and Y. Wang, “Supplementation with Lactobacillus kefiranofaciens ZW3 from Tibetan Kefir improves depression-like behavior in stressed mice by modulating the gut microbiota," Food \& Function, vol. 10, no. 2, pp. 925-937, 2019.

[79] I. Semova, J. D. Carten, J. Stombaugh et al., "Microbiota regulate intestinal absorption and metabolism of fatty acids in the zebrafish," Cell Host \& Microbe, vol. 12, no. 3, pp. 277-288, 2012.

[80] F. Bäckhed, J. K. Manchester, C. F. Semenkovich, and J. I. Gordon, "Mechanisms underlying the resistance to diet-induced obesity in germ-free mice," Proceedings of the National Academy of Sciences of the United States of America, vol. 104, no. 3, pp. 979-984, 2007.

[81] R. Rastmanesh, "High polyphenol, low probiotic diet for weight loss because of intestinal microbiota interaction," Chemico-Biological Interactions, vol. 189, no. 1-2, pp. 1-8, 2011.

[82] C.-J. Chang, C.-S. Lin, C.-C. Lu et al., "Erratum: Corrigendum: Ganoderma lucidum reduces obesity in mice by modulating the composition of the gut microbiota," Nature Communications, vol. 8, no. 1, 2017.

[83] A. Gori, C. Tincati, G. Rizzardini et al., "Early impairment of gut function and gut flora supporting a role for alteration of gastrointestinal mucosa in human immunodeficiency virus pathogenesis," Journal of Clinical Microbiology, vol. 46, no. 2, pp. 757-758, 2008.

[84] M. L. Wong, A. Inserra, M. D. Lewis et al., "Inflammasome signaling affects anxiety- and depressive-like behavior and gut microbiome composition," Molecular Psychiatry, vol. 21, no. 6, pp. 797-805, 2016. 